\title{
Allariz. La puesta en valor del patrimonio natural y cultural como elemento clave en su desarrollo
}

\author{
María Garrote Recarey ${ }^{a}$
}

${ }^{a}$ Universidade da Coruña, mgarroterecarey@gmail.com

\begin{abstract}
Resumen
La villa de Allariz es una pequeña joya patrimonial del pueblo gallego. Al contrario de lo que pasó con otras villas gallegas, su casco histórico, bañado por las aguas del río Arnoia, permaneció inalterado a lo largo del tiempo. En él se pueden apreciar huellas de su pasado medieval como residencia real, su esplendor señorial o su reconversión en ciudad protoindustrial, con múltiples curtidurías en los márgenes del río.

A pesar de su declaración como Conjunto Histórico-Artístico en 1971, la villa y su entorno se encontraban a finales de los años ochenta del siglo pasado en un estado de total abandono. En la década siguiente, después de una enorme presión vecinal, el gobierno municipal aprobó el Plan Especial del Casco Histórico. La creación de una oficina de rehabilitación y una escuela-taller, permitió la recuperación de equipamientos y espacios públicos, lo que le valió el Premio Europeo de Urbanismo en 1994.
\end{abstract}

El objetivo de mi ponencia será analizar los distintos planes llevados a cabo en las últimas décadas que permitieron recuperar su aspecto histórico y su perímetro natural, de forma que en la actualidad la ciudad emplea su patrimonio como motor económico.

Palabras clave: patrimonio, naturaleza, cultura, urbanismo, desarrollo, rehabilitación, paisaje, turismo.

\begin{abstract}
The village of Allariz is a small heritage jewel of the Galician territory. Unlike what has happened to other Galician villages, its historic centre, crossed by the river Arnoia, has remained unchanged throughout history. We can see traces of its medieval past as a royal residence, its stately magnificence or its transformation in a protoindustrial city, with multiple tanneries on the bank of the river.

Despite its declaration as a Historic-Artistic Site in 1971, the village and its surroundings were being abandoned at the end of the last century. In the following decade, after an enormous neighbours demands, the local government approved the Special Plan for the Historic District. The creation of a Restoration Department and a workshop, allowed for the recovery of equipment and public spaces, which was the key for win the European urban and regional planning Award.

The objective of this paper will be to analyze the different plans carried out over the last years that have permitted the recovery of its historical aspect and its natural perimeter, so that at present, the town's heritage has become its economic engine.
\end{abstract}

Keywords: heritage, nature, culture, urbanism, development, restoration, landscape, tourism. 


\section{El patrimonio alaricano y el río Arnoia}

La villa de Allariz se localiza en un valle de la provincia de Ourense, a $20 \mathrm{Km}$ de su capital. Es cabeza del término municipal del mismo nombre que actualmente tiene una población de en torno a 6.000 habitantes. La villa, que desde su origen fue un importante cruce de caminos, está bañada por el río Arnoia, que inicialmente sirvió como barrera defensiva y acabó convirtiéndose en símbolo de su recuperación.

El patrimonio alaricano se conformó a lo largo de los siglos, destacando especialmente tres épocas. En primer lugar la Edad Media, en la que la villa se convirtió en residencia real para los reyes leoneses. En ella se eregía un castillo, destacado en la defensa de la frontera con el recién creado reino de Portugal, que se completaba con un entramado defensivo de murallas y barbacanas. En este periodo se construyeron varias iglesias románicas, hoy conservadas.

Un segundo momento fue el asentamiento en la villa de familias nobles e hidalgas, que crearon una arquitectura particular, los palacios urbanos, que destacaban sobre el caserío uniforme de la villa. En este momento también se produjo la expansión del convento de Santa Clara, situado extramuros y que había sido fundado por doña Violante, mujer de Alfonso XII.

Por último, durante el siglo xix se produjo el desarrollo de la industria del cuero en la villa. El curtido de pieles siempre había estado muy presente -según el Catastro de Ensenada, la profesión de zapatero era la más popular- y en este momento se pasa de una tarea artesana a fábricas con trabajadores asalariados. Puesto que eran consideradas insalubres, estas curtidurías se situaban extramuros a lo largo del río Arnoia, en donde también se localizaban pequeñas aceñas y molinos harineros.

El cierre de la última curtiduría en los años sesenta del siglo pasado y la progresiva pérdida de población provocó el abandono de los edificios fabriles y los molinos hidráulicos, a la que hubo que sumar la polución del cauce del río debido principalmente a vertidos nocivos, arrojados sin el tratamiento adecuado. A causa de la dejadez del gobierno municipal en lo referente a la degradación del río, surgió en la villa, en enero de 1989 (El 'comité en defensa do Arnoia' [...], 1989), la plataforma Comité de Defensa do río Arnoia, con la misión de denunciar la situación, así como de elaborar propuestas para su recuperación.

La situación se agravó unos meses después, momento en que aparecieron un centenar de truchas muertas en el cauce del cada día más contaminado Arnoia (Aparecieron muertas [...], 1989). La reiterada negativa del alcalde a convocar el pleno para tratar la situación del río fue el detonante para el encierro de un centenar de vecinos en el edifício municipal el día 11 de agosto (Vecinos de Allariz [...], 1989), acción que se vio acompañada de otras: manifestaciones, paros y trabajos voluntarios con los que buscaban dar a conocer las que consideraban como carencias de la villa.

Después de varios meses de encierro, el 20 de noviembre, la denominada asamblea popular de veciños de Allariz forzó la dimisión del alcalde, eligiéndose días más tarde a Anxo Quintana -portavoz del Bloque Nacionalista Galego y de la propia asamblea vecinal- nuevo alcalde por unanimidad.

Nada más acceder al poder, el nuevo equipo de gobierno puso en marcha las propuestas que consideraban urgentes, como la creación de un parque móvil municipal o la mejora del alumbrado. Aunque si un tema ocupó al equipo de gobierno fue, por supuesto, el problema del saneamiento del Arnoia, pues el río se había convertido en el protagonista de la rebelión del pueblo, cuyo ejemplo se extendió por otros pueblos gallegos como Verín, Lalín o Monterrei (Vecinos de Muiños amenazan [...], 1991).

\section{Primeras actuaciones. El germen del Allariz turístico}

Una vez satisfechas las necesidades básicas de la población, tales como recogida de basuras, alumbrado o agua, comenzaron a llevarse a cabo una serie de actuaciones que serían el germen de las ejecutadas en las posteriores normas y planes. Para poder afrontar la rehabilitación de algunas de las piezas clave de la villa se creó una escuela-taller, idea 
que ya se había puesto en práctica con resultados desiguales en otras localidades como Ribadavia ${ }^{1}$. La escuela Ziralla, que fue inaugurada en enero de 1991 con una duración prevista de tres años, constaba de seis módulos: cantería, albañilería, carpintería, jardinería, forja y animación sociocultural, este último encargado de la organización de actividades de promoción de la villa a través de su valor histórico, cultural y paisajístico. Para ponerlo en marcha se contó con la financiación del INEM y del Fondo Social Europeo.

Por la escuela pasaron cincuenta y seis alumnos y siete profesores, con el objetivo de la "recuperación integral del casco antiguo de le villa y de los espacios naturales con especial atención a los márgenes del río Arnoia" (El alcalde de Allariz [...], 1990). Eran los jóvenes desempleados de la villa los que participaban en la recuperación de su propio patrimonio. Entre los proyectos llevados a cabo por la escuela estaban la recuperación del molino do Burato como museo etnográfico, la remodelación del campo da Barreira o la reconstrucción del centro social O Portelo (El ejemplo alaricano, 1993). Después de tres años de trabajo la escuela se clausuró en diciembre de 1993, destacando el gran volumen de obra realizada y con la aspiración de crear una nueva escuela para finalizar tareas comenzadas o proyectadas.

Otra de las actuaciones puntuales promovidas para la mejora del casco histórico y el río fue la reforma de una antigua fábrica de curtidos donada por un particular (Un particular dona [...], 1990). Este espacio, conocido como La Fábrica, fue inaugurado en mayo de 1991, y contaba con sala de exposiciones, cafetería y almacén para barcas y piraguas, así como embarcadero. La Xunta de Galicia, por su parte, adquirió la antigua cárcel alaricana, con el objetivo de convertirla en Casa de Cultura. El edificio fue inaugurado en septiembre de 1991, destacando el salón de actos y la biblioteca municipal.

En octubre de 1992 se firmó un convenio para habilitar un local en las dependencias del Convento de Santa Clara con el fin de ubicar en él un Museo de Arte Sacro, que quedaría enmarcado en el sistema público de museos de la comunidad autónoma. Entre las piezas que custodiaba el convento y que hoy se exhiben, las de mayor valor son la Virxe Abrideira y la Cruz de Cristal.

Por último destacar que, basándose en el proyecto de recuperación del río, elaborado en su momento por el Comité, se planteó la recuperación del entorno cuya primera fase consistió en la limpieza de los márgenes, la recomposición de las compuertas de varias presas y la construcción de colectores de saneamiento ${ }^{2}$.

\section{Normas Subsidiarias de 1994}

"El futuro planeamiento de Allariz deberá tener como norte el establecimiento de un modelo que sin renunciar a una auténtica modernidad, preserve los valores esenciales del territorio -naturales y artificiales-y facilite una explotación racional de los recursos tradicionales, como único medio de alcanzar una mejora de la calidad de vida para el conjunto de su población, tanto urbana como rural.” (Portela y Pino, 1992).

Allariz, que había sido definida en las Normas Subsidiarias y Complementarias de la Provincia de Ourense como un municipio con un alto grado de complejidad urbanística (Resolución de 14 de mayo de 1991 por la que se ordena la publicación de las normas complementarias y subsidiarias de planeamiento de las provincias de A Coruña, Lugo, Ourense y Pontevedra) compuesto por más de ochenta núcleos de población, no contaba con un planeamiento propio. Sin embargo, la creación de un plan comenzó pronto a verse como un instrumento esencial para la ordenación del territorio.

Una vez hubo tomado posesión de sus cargos, el nuevo equipo de gobierno comenzó a trabajar en él, actuando desde tres frentes. En primer lugar la administración, que convocó un Concurso Público en noviembre de 1991 con el fin de adjudicar la redacción de las Normas. En segundo lugar, el equipo técnico ganador del Concurso en enero de 1992,

\footnotetext{
${ }^{1}$ Cuando se creó en Allariz la escuela-taller se le dotó de entidad jurídica propia, convirtiéndose en un ente autónomo, para evitar problemas como los que habían surgido en Ribadavia entre el nuevo equipo de gobierno y la escuela.

${ }^{2}$ Las obras de los colectores del margen izquierdo eliminarían el 70\% de las aguas residuales vertidas desde el centro. Trabajan de noche para sanear el río Arnoia y evitar cortes de agua a los usuarios de Allariz (1990).
} 
Consultora Galega S.L. Por último los ciudadanos, cuyas opiniones y sugerencias fueron tomadas en cuenta desde las primeras etapas del proyecto.

La organización de la participación ciudadana, tal y como se recoge en las Normas, se desarrolló en un primer momento a través de entrevistas y encuestas para determinar la percepción de los habitantes sobre su municipio ${ }^{3}$. A medida que avanzaba la redacción, se divulgaba por diferentes medios y unos quinientos vecinos eran atendidos de manera individualizada (Portela y Pino, 1992). De las sugerencias recogidas en este periodo más del $80 \%$ tuvieron plena aceptación y más del 90\% aceptación parcial en la redacción final (Portela y Pino, 1992).

El objetivo general que debía marcar el rumbo de lo que debía ser el urbanismo de la villa, era la búsqueda de la mayor calidad de vida posible, consistente en la mejora de la habitabilidad y no sólo el incremento del nivel de vida. Esta premisa estaría presente sobre todo en el diseño de dotaciones y en la preservación del medio ambiente.

Además se planteaba la necesidad de proteger el patrimonio natural y el patrimonio histórico artístico, etnográfico y cultural de la villa. De esta manera se conservaban los medios económicos de los que vivían los núcleos rurales explotación de recursos agropecuarios y forestales-, creándose asimismo oferta equipamental -nuevas infraestructuras, edificios hosteleros, ... - construidos siempre con respeto y sensibilidad, para favorecer el desarrollo del sector terciario en la población urbana.

Centrándonos en la población urbana, los equipamientos proyectados eran de carácter esencialmente sociocultural, ligados a la propia labor de recuperación del patrimonio histórico. Las construcciones se proponían diseminadas por todo el territorio y su oferta era diversa. Se advertía de la importancia que tendría complementar esta oferta cultural con otras actividades que posibilitaran la autosuficiencia de estos espacios (Fig. 1).

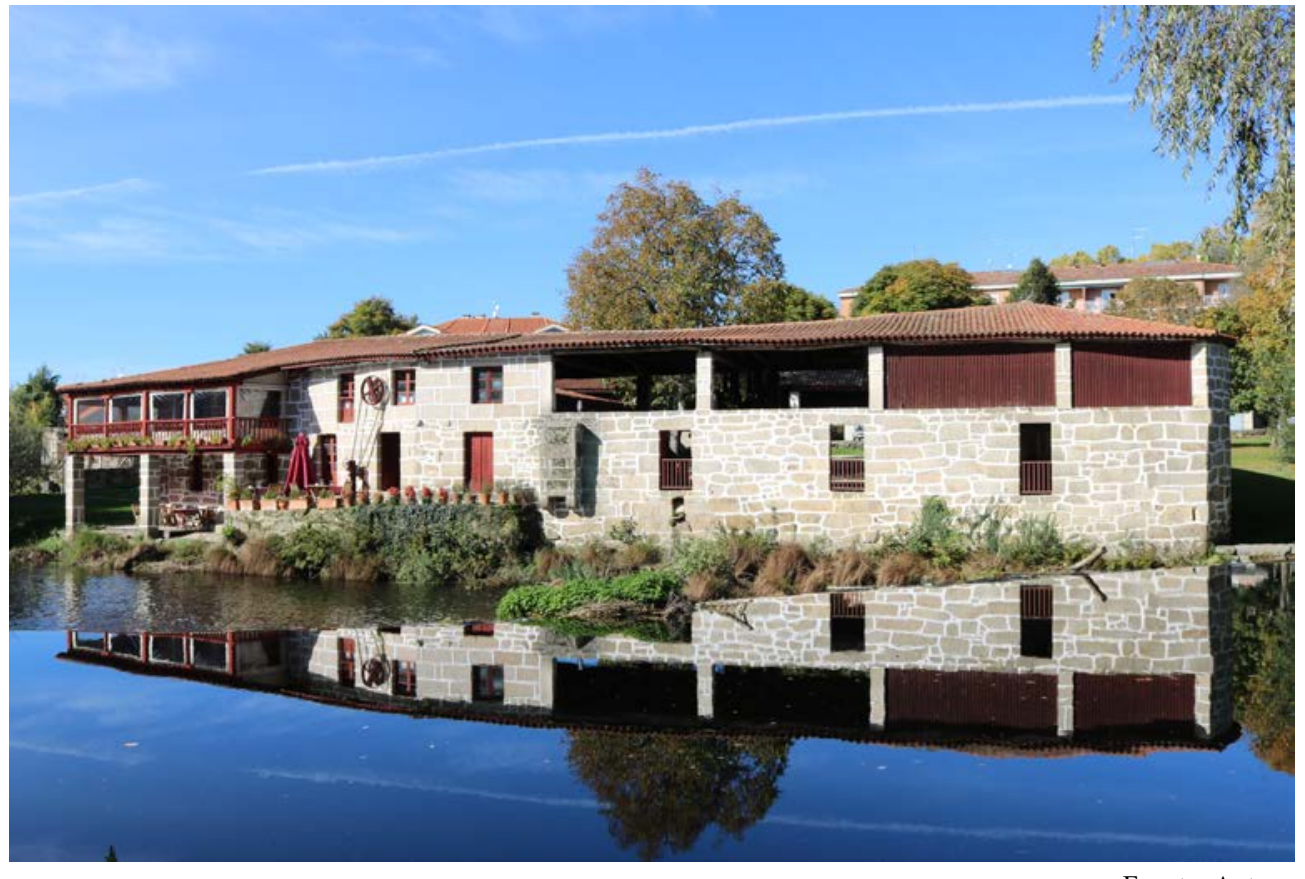

Fuente: Autora

Fig. 1 Antigua fábrica Nogueiras, hoy Museo del Cuero

Para cubrir las demandas de la población más joven y atraer al municipio a este estrato de la sociedad, se hacía imprescindible la mejora de los equipamientos deportivos existentes, trasladando aquellos mal ubicados -como el campo de fútbol- así como el mantenimiento de las tres zonas escolares existentes cada una de ellas destinada a un tipo de enseñanza.

\footnotetext{
${ }^{3}$ Las encuestas recogen preguntas como "qué cosas le gustan más de su parroquia", "qué cosas le gustan más de su ayuntamiento", "qué le gusta menos de su parroquia o de su ayuntamiento" o "qué echa de menos en su lugar o en su parroquia". Portela Jardón, C. y Pino Vicente, D. (1992), Normas Subsidiarias Municipales de planeamiento de Allariz. Memoria Informativa, Anexo 3.
} 
Se remarcaba asimismo la futura importancia que tendría el sistema de espacios verdes y zonas libres. Se proponía completar la oferta presente en el entorno del río, así como acondicionar la zona del Campo da Barreira y la del castillo. Estos tres elementos -Arnoia, Barreira y Castelo- se convertían así en los hitos más significativos de la villa.

Por último, la clasificación del suelo urbano proponía consolidar un modelo territorial que tuviera en cuenta el nuevo papel turístico del espacio a la vez que solucionaba los problemas de comunicación presentes en el núcleo.

Este instrumento básico de ordenación territorial aprobado de manera definitiva en marzo de 1994, se completaría en los siguientes años con un Plan Especial de ordenación del Casco Histórico, actuación que era entendida como pieza clave para el futuro de la villa:

“Armonía entre Naturaleza y Artificio es la constante paisajística más destacable de Allariz. [...] Naturaleza e Historia constituyen en Allariz no sólo los signos de identidad de un pueblo, sino también un importantísimo recurso económico, pues son muchos los que acuden a esta tierra, precisamente para poder disfrutar de ellas." (Portela y Pino, 1992).

\section{Plan Especial del Casco Histórico de Allariz de 1995}

Las Normas Subsidiarias planteaban por vez primera la necesidad de redactar el Plan Especial del Casco Histórico de Allariz (PECHA), que ya había sido declarado Conjunto Histórico-Artístico en 1971 (Decreto 1319/1971, de 20 de mayo, por el que se declaran conjuntos histórico-artísticos varias zonas de la villa de Allariz, 1971, p.10149). Con el fin de iniciar su redacción se convocó un concurso público en octubre de 1992, que ganó de nuevo Consultora Galega S.L. El equipo de redacción estaba dirigido por el arquitecto Cesar Portela, coordinado por el sociólogo urbanista Daniel Pino y aglutinaba a especialistas de diversas áreas, especialmente arquitectos e historiadores.

Para delimitar el ámbito del conjunto que se iba a incluir en el futuro PECHA se partió de las zonas que habían sido acotadas por la declaración de 1971, que comprendían aproximadamente $289.000 \mathrm{~m}^{2}$.

La grave crisis económica que había sufrido Allariz a mediados del siglo XX, que culminó con el cierre de las curtidurías y la emigración de buena parte de los alaricanos, había reducido en 4.000 habitantes la población del ayuntamiento. En 1993 vivían en el casco unas 850 personas, la maypría de edad avanzada y bajo poder adquisitivo. Sin embargo, esta situación tuvo su parte positiva, pues a pesar de no haber contado con una protección específica, la falta de recursos económicos para actuar sobre el casco permitió que llegara hasta finales del siglo XX en buen estado de conservación, mejor que otros cascos históricos de características parecidas.

El planteamiento desarrollado en el PECHA partía de la idea de que la clave para la recuperación económica de la villa era la recuperación de su propio patrimonio. Los recursos, la materia prima que permitiría este crecimiento, estaban en el casco histórico o en su entorno inmediato: el río Arnoia. Naturaleza e historia quedaban así convertidas en los motores económicos de la recuperación de la villa.

No se trataba de congelar la villa en el tiempo, se trataba de dotar a las construcciones antiguas de nuevos usos acordes con las necesidades del momento, complementándolos con atracciones turísticas de calidad, buscando un equilibrio entre la conservación y recuperación del patrimonio y la mejora de las condiciones de vida de la población. Estas ideas quedaban recogidas en la redacción de los objetivos generales del Plan, que son los siguientes:

1. Búsqueda del desarrollo integral de Allariz, tratando el casco histórico como complementario al resto del territorio.

2. Conservación del patrimonio arquitectónico y urbanístico, que se estudiaría por medio de inventarios y catálogos, fijándose medidas a adoptar para su recuperación, mejora y puesta en valor.

3. Integración del área que conformaba el conjunto histórico en el tejido urbano y en el contexto funcional de la villa, diversificando los usos compatibles con su escala.

4. Respeto por la estructura urbana y mantenimiento de la escala y proporciones en las intervenciones llevadas a cabo, de manera que las formas propuestas debían armonizar con el entorno del casco urbano. 
5. Creación de nuevos valores, tanto recuperando los que se habían perdido como diseñándolos de nuevo, todo ello respondiendo a unas necesidades, mejorando la calidad de vida de la población y añadiendo valor al casco.

Las directrices generales establecidas por el ayuntamiento para la redacción del Plan y aplicadas a cada una de las diversas piezas a proteger, partían principalmente de tres puntos (Portela y Pino, 1993):

- Protección del patrimonio arquitectónico y cultural.

- Mejora de las condiciones de vida de la población.

- Integración de la ciudad histórica en el tejido urbano.

La conciencia social se entendía como un punto de enorme importancia a la hora de acometer la mejora del casco, igual que había pasado con las Normas Subsidiarias. A pesar del envejecimiento y de su bajo poder adquisitivo, la población presentaba una visión muy favorable hacia la recuperación y puesta en valor del patrimonio de la villa. Todo el proceso volvió a ser permeable a la participación de la ciudadanía, que pudo acceder a la información desde el inicio y plantear sus dudas en diferentes momentos de la redacción. También se llevaron a cabo encuestas a las familias, tanto a las que residían habitualmente en el casco histórico, como a las que lo hacían de de manera esporádica, obteniendo una valiosa información sobre la opinión que tenía la población respecto a las reformas, sus necesidades y su nivel socioeconómico. Por otro lado, se encuestó también a los propietarios de bajos comerciales del casco, haciendo un estudio minucioso de sus locales, clasificándolos según su uso.

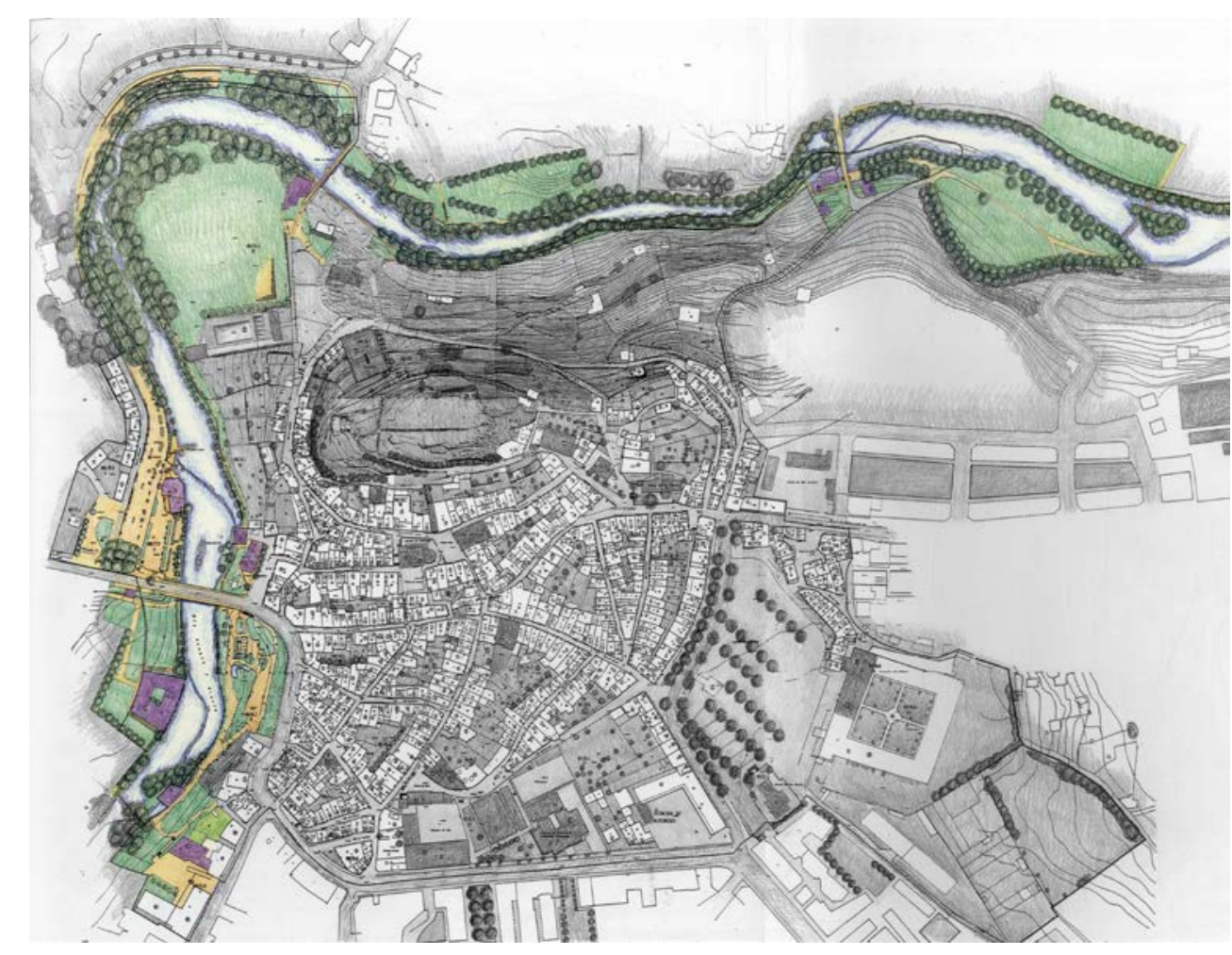

Fuente: Dibujo modificado por la autora

Fig. 2 Proyecto de intervención en el PEDRA, 1994. Dirección Xeral de Patrimonio Cultural de Galicia, exp. 1994/012 y VV.AA. (1997). Oficina Municipal de Rehabilitación do Casco histórico de Allariz. Memoria resumen dun ano de actividade. Obradoiro: revista de arquitectura y urbanismo, 26, 31

La rehabilitación del casco propuesta en el Plan tenía un elevado coste económico. Sin embargo, al tratarse de un Bien de Interés Cultural, Allariz tenía derecho a recibir ayuda de diversas administraciones, para cuya gestión el ayuntamiento creó una Oficina Municipal de Rehabilitación. Esta oficina, todavía hoy en funcionamiento, ofrece entre otros servicios diagnóstico y asesoramiento a los vecinos que deseen rehabilitar sus viviendas y tramitación de las ayudas a las que tienen derecho. 
Para poder optar a estas ayudas, el ayuntamiento debía fijar el Área de Rehabilitación Integrada (ARI), que incluía a la totalidad del casco histórico consolidado. Sumando las ayudas ofrecidas por el Ministerio y las de la Xunta de Galicia se podía cubrir hasta un $40 \%-50 \%$ del coste total de la rehabilitación, mientras que se ofrecía la posibilidad de financiar el resto con préstamos a bajo interés (Portela y Pino, 1993). Dada la cuantía de las ayudas, como es de suponer, su gestión por parte de la Oficina Municipal de Rehabilitación resultó de crucial importancia para el éxito de la rehabilitación de propiedades privadas.

La recuperación de equipamientos comunes y espacios públicos, que había dado comienzo en los años anteriores, partía de la premisa de la rehabilitación de piezas y espacios significativos del patrimonio alaricano, que pasaban a formar parte de su oferta equipamental y donde se desarrollaban actividades de interés tanto para la población como para futuros visitantes. Los equipamientos eran entendidos como centros de polaridad urbana, por lo que su potenciación y mejora era imprescindible para mantener con vida al casco.

En cuanto a zonas verdes y espacios libres el Plan daba especial importancia a tres grandes áreas. El primer gran espacio presente en la villa era la agreste roca donde se había asentado en su día el castillo, que se acondicionaría como mirador, recuperándose su entorno. También se indicaba la necesidad de un plan específico para el campo da Barreira, el gran espacio urbano de la villa, que se mantendría vacío disponible para albergar ferias y fiestas puntuales.

Por último, el llamado Parque Etnográfico Do Río Arnoia (PEDRA), la obra paradigmática del Plan, que pretendía unificar el sistema de espacios verdes que discurrían paralelos al eje del Arnoia (Fig. 2), enlazando parques y zonas verdes ya construidos y conectando ambos márgenes mediante pasarelas peatonales. El visitante del Parque realizaba un recorrido por la historia de la villa visitando una serie de elementos culturales: el museo del cuero, el museo etnográfico y el desaparecido museo del tejido -hoy reconvertido en mercado de la Biosfera-. Estos espacios se completaban con elementos de hostelería: un hotel en la fábrica Torre Lombarda y restaurantes en la Fábrica de Vilanova, el molino do Burrelo. Todos ellos se recuperaron siguiendo los principios de respeto hacia los valores históricos y etnográficos que poseían.

\section{Conclusiones}

El casco histórico alaricano es uno de los de mayor interés de Galicia, así como también de los mejor conservados. Tres hechos fueron, en mi opinión, clave para el éxito de su recuperación. En primer lugar la creación de una escuela-taller, financiada por el INEM y el Fondo Social Europeo, que pudo afrontar la rehabilitación de algunas de las piezas más importantes de la villa. En segundo lugar, la creación de una Oficina Municipal de Rehabilitación, que todavía hoy ofrece asesoramiento a la vez que controla los proyectos y ayuda a los vecinos en la tramitación de las imprescindibles subvenciones. Por último la creación de REATUR, empresa pública dedicada a la rehabilitación y el fomento del turismo rural, encargada entre otras cosas de desarrollar el comercio en la villa, con acciones como el establecimiento de outlets en el interior del casco.

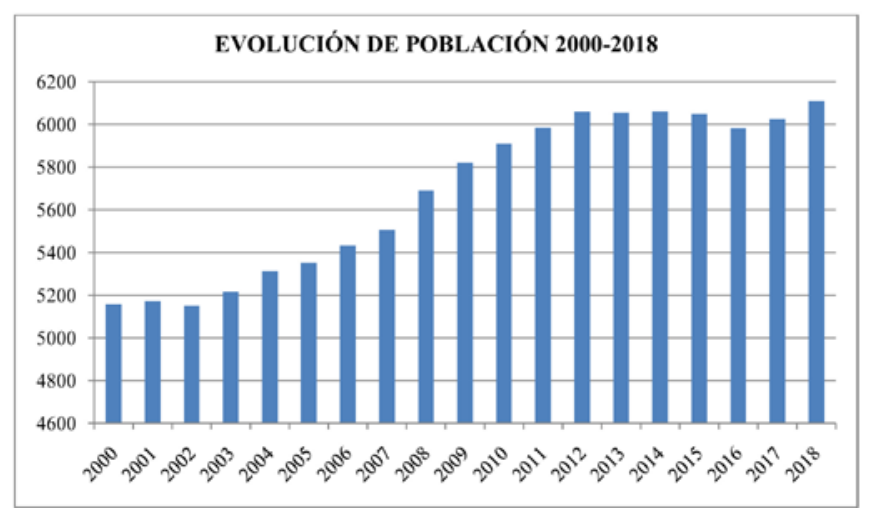

Fuente: Autora a partir de datos del Instituto Nacional de Estadística

Fig. 3 Población del municipio de Allariz entre 2000 y 2018. Gráfico de la autora a partir de datos del Instituto Nacional de Estadística 
El trabajo de recuperación realizado en la villa obtuvo en 1994 el Premio Europeo de urbanismo y planificación regional. A partir de la aprobación del PECHA se ha llevado a cabo, con muy buen criterio, la rehabilitación de ciertas piezas y espacios, que se convirtieron de inmediato en recurso económico, potenciando un turismo de calidad. En la actualidad la villa cuenta con una interesante red de museos y se han recuperado asimismo tradiciones y fiestas que complementan la oferta. La situación demográfica de la villa presenta una mejoría desde el año 2000, sin duda gracias a la aplicación de estas medidas (Fig. 3).

Naturaleza y Patrimonio son el binomio sobre el que hoy en día se sustenta la economía alaricana. Sus recursos naturales y su riqueza patrimonial son las materias primas que permiten su crecimiento. Esta fue la premisa propuesta desde su inicio por los planeamientos municipales que se ha convertido en la clave de su recuperación.

\section{Referencias}

Aparecieron muertas un centenar de truchas en la Arnoia a su paso por Allariz (1989, 3 de agosto). La Voz de Galicia, p. 21.

Boletín Oficial del Estado. (1971). Decreto 1319/1971, del 20 de mayo, por el que se declaran conjuntos histórico-artísticos varias zonas de la villa de Allariz (Orense). Publicado en BOE, 148, 22 junio 1971, 10149.

Diario Oficial de Galicia. (1991). Resolución de 14 de mayo de 1991 por la que se ordena la publicación de las normas complementarias y subsidiarias de planeamiento de las provincias de A Coruña, Lugo, Ourense y Pontevedra. Publicado en DOG, $\mathrm{n}^{\circ}$ 116, 19 junio 1991.

El alcalde de Allariz presentó al Inem el proyecto de una escuela taller que costará cerca de 300 millones de pesetas (1990, 30 de marzo). La Voz de Galicia, 31.

El “comité en defensa do Arnoia” desarrollará una campaña informativa (1989, 22 de enero). La Voz de Galicia, 37.

El ejemplo alaricano (1993, 31 de diciembre). La Voz de Galicia, s.n.

Instituto Nacional de Estadística. Recuperado el 13 de marzo de 2020 de: https://www.ine.es/FichasWeb/Welcome.do

Portela, C. (Dir.) y Pino, D. (Coord.). (1992). Normas Subsidiarias Municipais de Planeamento de Allariz.

Portela, C. (Dir.) y Pino, D. (Coord.). (1993). Plan Especial do Casco Histórico de Allariz.

Un particular dona una finca al Ayuntamiento de Allariz con la única condición de que se utilice (1990, 17 de febrero). La Voz de Galicia, 23.

Vecinos de Allariz continuarán este fin de semana el encierro iniciado ayer en la Casa Consistorial (1989, 15 de agosto). La Voz de Galicia, 57.

Vecinos de Muiños amenazan con 'otro Allariz' si no se respeta el resultado electoral que da la mayoría al PSOE (1991, 6 de junio). La Voz de Galicia, 42.

VV. AA. (1997). Oficina Municipal de Rehabilitación do casco histórico de Allariz. Memoria resumen dun ano de actividade. Obradoiro: Revista de arquitectura y urbanismo, 26, 29-34. 